\title{
Design and Development of Water Quality Monitoring System Based on Wireless Sensor Network in Aquaculture
}

\author{
Mingfei Zhang, Daoliang Li, Lianzhi Wang*, \\ Daokun Ma, and Qisheng Ding \\ College of Information and Electrical Engineering, \\ China Agricultural University, \\ Beijing, China \\ zmf1985@126.com, dliangli@cau.edu.cn, ndjsj862@cau.edu.cn
}

\begin{abstract}
This paper presents a system framework taking the advantages of the WSN for the real-time monitoring on the water quality in aquaculture. We design the structure of the wireless sensor network to collect and continuously transmit data to the monitoring software. Then we accomplish the configuration model in the software that enhances the reuse and facility of the monitoring project. Moreover, the monitoring software developed to represent the monitoring hardware and data visualization, and analyze the data with expert knowledge to implement the auto control. The monitoring system has been realization of the digital, intelligent, and effectively ensures the quality of aquaculture water. Practical deployment results are to show the system reliability and real-time characteristics, and to display good effect on environmental monitoring of water quality.
\end{abstract}

Keywords: WSN, Water quality monitor, Zigbee, Control, System integration.

\section{Introduction}

China as a huge fishery country produces a great quantity of aquaculture every year in the world. As all known, water quality is very important in aquaculture, so the real-time monitoring and early warning studies on the water environment have been an essential part of aquaculture. At present, most domestic water quality monitoring are still using manual methods. The detection process involved in sampling, sample transportation and preservation, laboratory data measured etc., is a complex but associated system. Any error of the steps will affect the results of the final data. The traditional artificial method as regular or irregular sampling and monitoring is waste of time, inefficient, and difficult to objectively reflect variation rules and facts. It has been unable to meet

* Corresponding author: WANG Lianzhi, master instructor. Research: Application of artificial intelligence. 
current water quality monitoring needs. Along with the quickly development in communication technology and sensor technology, it accelerates the remote monitoring and automatic water quality monitoring process. The real-time water quality monitoring system based on wireless sensor network due to its convenience, real-time, accurate characters, has been get researchers more and more attentions[6-12].

\section{System Architecture Based on WSN (Wireless Sensor Network)}

The process to monitor water quality environment includes data acquisition, data transmission, data preservation and decision-making, which is involved with software and hardware comprehensive integration, so the excellent architecture becomes the top priority to the water quality monitoring system as a composite system.

\subsection{Introduction to WSN}

WSN (Wireless sensor network) is composed of data acquisition node, wireless transmission network and information processing center. Data acquisition nodes integrate sensors, data processing module and communication module. Through the communication protocol, the nodes form a distributed network. Then the network transmits the optimization data to information processing center. The system adopts Zigbee based on IEEE 802.15.4 communication protocol. Zigbee is a wireless intercommunication technique that has low transmitting rate and low cost advantages. It can be embed in devices, is particularly suitable for industrial control, aquaculture water quality monitoring, wireless sensor networks and smart devices such as the widely distributed applications [1-4].

Wireless network monitoring system includes the major technologies:(1)Wireless communication technology $-2.4 \mathrm{GHz}$ short range communication and GPRS communication, wireless signals cover $3 \mathrm{~km}$ range; (2)Embed control technology-intelligent information acquisition and control; (3)Wireless network technology-network routing layer, communication caching, self-diagnosis and maintenance technology; (4)Energy manage technology-low power consumption and energy management.

Wireless sensor networks are widely used throughout intelligent transportation, environmental protection, public safety, peace at home, smart fire alarm, industrial monitoring, elderly care, personal health, floriculture, food traceability, enemy detection and intelligence gathering, and other fields ${ }^{[5]}$. Particularly in the areas of agriculture and rural information, WSN can be more widely available, such as: precision agriculture-the precise application of sensor technology, intelligent expert management system, remote monitoring and remote sensing systems, bioinformatics and diagnostic systems, food safety traceability system.

\subsection{Water Quality Monitoring System Architecture Applied WSN}

Based on WSN, the water quality monitoring system uses three-layer structure: data acquisition layer, data transport layer and application layer to establish its architecture. The architecture as figure 1 shows: 


\begin{tabular}{|c|c|c|}
\hline \multicolumn{3}{|l|}{ Application layer } \\
\hline Monitor software & Control commands & \\
\hline Data & Commands & \\
\hline \multicolumn{3}{|l|}{ Data transport layer } \\
\hline Zigbee,Gateway,GPRS & \multicolumn{2}{|c|}{ Zigbee, Gateway,GPRS } \\
\hline Data & Commands & \\
\hline Data acquisition layer & Data control layer & \\
\hline Sensors, RFID & contro1 valves, windows & \\
\hline
\end{tabular}

Fig. 1. System architecture based on WSN

In the data acquisition layer, the water quality monitoring system uses WSN which is formed by a large number of tiny sensor nodes through wireless self-organizing way. The sensors obtain data in the network include PH, water level, water temperature, DO (dissolved oxygen) sensor. WSN integrates sensor, MEMS and wireless communication technologies, can real-time perception and handle object's data information in network range, then send to the users. It has many excellent characters, such as large cover range, remote monitor, high monitoring precision, quick deployment and low cost. Through using WSN technology, the system can effectively monitor transport data and ensure the real-time data to be transported to the water quality monitoring software for analyzing and providing later control decisions.

In the data transport layer, there are two types of transmission methods: long-distance and short-distance data transmission. The system uses the GPRS to transport long-distance data, and uses the Zigbee to obtain the sensors' monitoring data, and then utilizes application software to manage the data and to make decisions to control devices. The real-time data through the sensors by Zigbee stored in the database to ensure users can query any time any sensor monitoring data information to analyze.

In the application layer, the water quality monitoring software is the main part. Through the soft ware, users can monitor the quality of the water all the time from the real-time data to ensure the water quality of the aquaculture. Water quality monitoring software utilizes the expert knowledge stored in the data and knowledge database together with the pretreatment data transported from sensors to make the decisions. Then the system decides whether to open or close the valve on relation device to increase or decrease the oxygen, water with the decisions. On-site users can receive early warning information from smart handheld device and depend on the decisions to operate. In order to facilitate user management, and data on water quality issues can occur in time to respond, the software also uses SMS early warning function. Off-site users can receive early warning information from PDA and other smart phones, and can send 
commands by SMS to control the valve on device. The users can not only get the current water quality environmental data, but also analyze the data in period to get the water environment trends to overall manage water quality.

The system collects temperature, $\mathrm{PH}$, dissolved oxygen sensor signals by sensor module, via wireless transport module transmit data, and combines with system software to achieve real-time monitoring and control. The system based on users demand, can monitor the water quality data at any time. It provides scientific evidences to automatic monitoring of water quality information, automatic control and intelligent management.

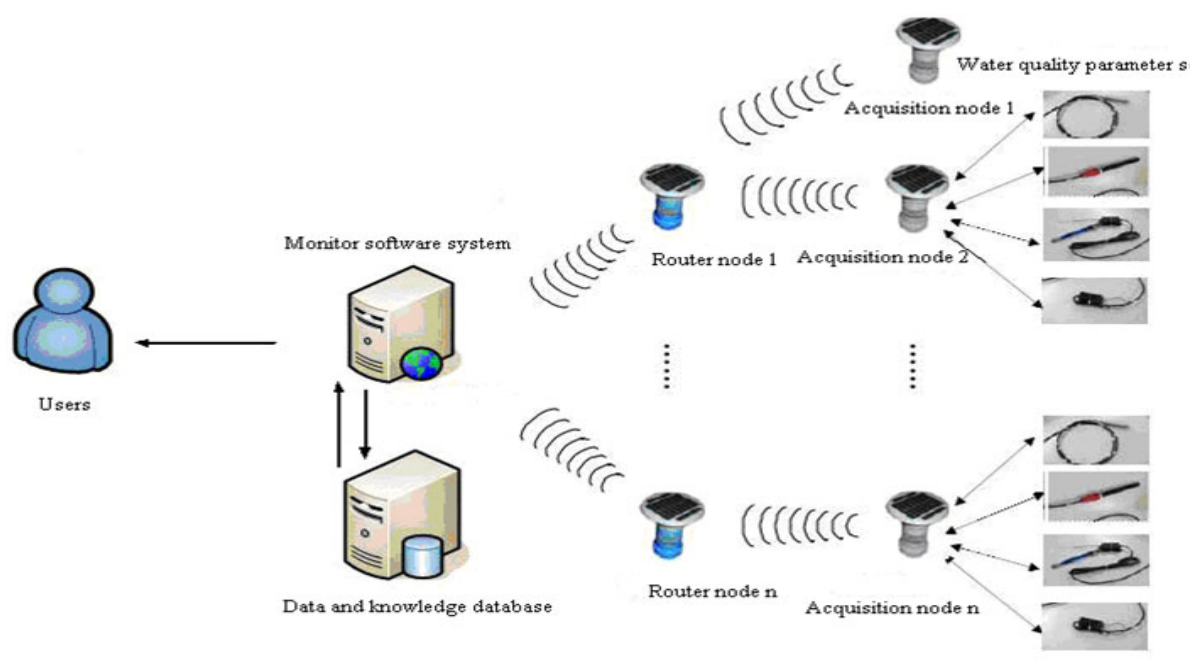

Fig. 2. System structure in application

\section{Water Quality Monitoring System Software Architecture and Function Models}

Water quality monitoring system software can pre-process, display and analyze the data from wireless sensor network, then make the corresponding decisions. Software system architecture and functional modules show as follows.

\subsection{Software Architecture}

The functions of water quality monitoring software in application layer are mainly the water quality data analysis and decision-making. The software development tool is Visual studio 2005, and develops, compile, debug in Windows XP. The software architecture based on .NET framework, using MVC structure, implements the separation of logic layer, display layer and data layer, which improved application scalability and reusability. The software architecture shows as the figure3. 


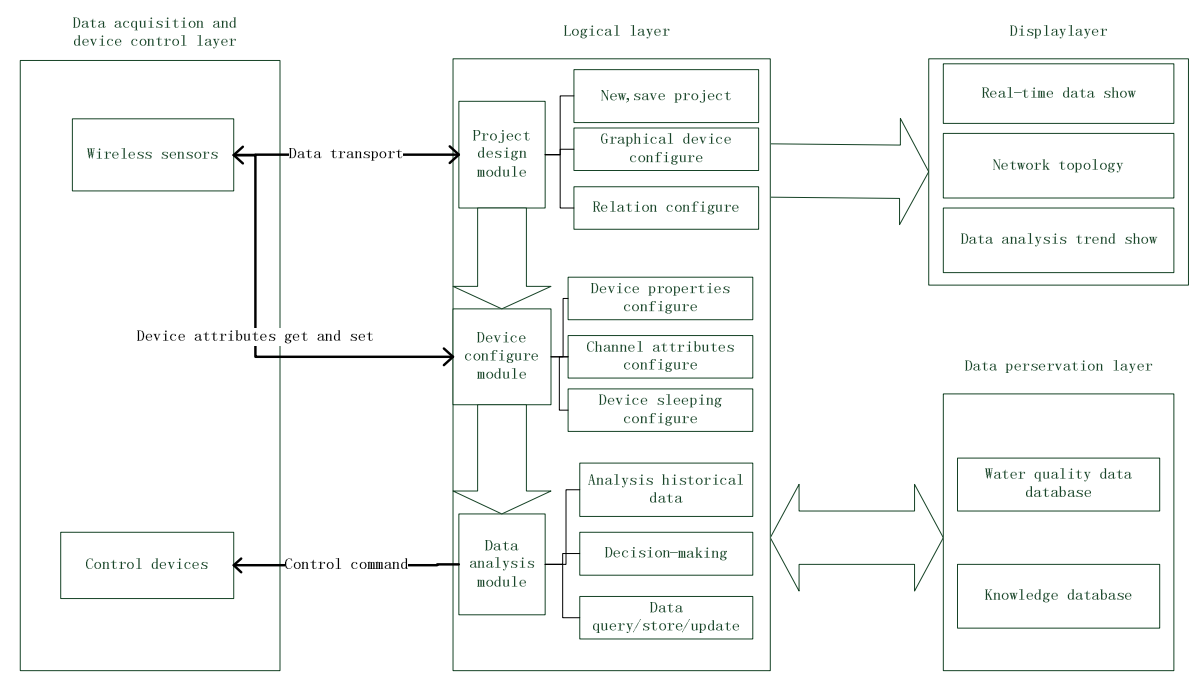

Fig. 3. Software architecture

In this architecture, the software hierarchical structure has three-layers: display layer, logic layer and data layer. Data acquisition and device control layer is related to the wireless sensor network hardware of the system architecture. The whole workflow process of the software is: firstly each sensor node in the wireless sensor network sends the water quality monitoring data to the water quality monitoring software; secondly in the design project, the users can see the real-time data by double-click the corresponding graphical device, and get the network topology. The user also can get the trends of one sensor data in a period or compare several sensors data in one comparison chart; thirdly according to the water quality data and device status, the users can add/delete device information, change the parameters of the device channel and set the sleeping status to the device for saving the battery power through the device configure module; fourthly through data analysis module, the software calls the knowledge from the knowledge database in the data layer to analyze the water quality data and decides to whether send control commands to the associated devices. The users can real-time monitor and manage aquatic water quality conveniently and efficiently by the various monitoring and control functions that the entire software modules work together to achieve.

\subsection{Software Function Modules}

The software main function modules include project design module, device configure module and data query/store/analyze module. Project design module through the user interface can display real-time data of water quality parameters in the monitoring region, over-limit alarm and historical data records; Device configure module uses communication protocol to communicate with the routing node, in order to achieve data 
transmission and device configuration aims, and in this module sensor network topology can be displayed to users; Data module uses database to implement the storage of the history monitoring data, and provides query and analysis operations to the users. Water quality monitoring software functions structure shows as figure 4 .

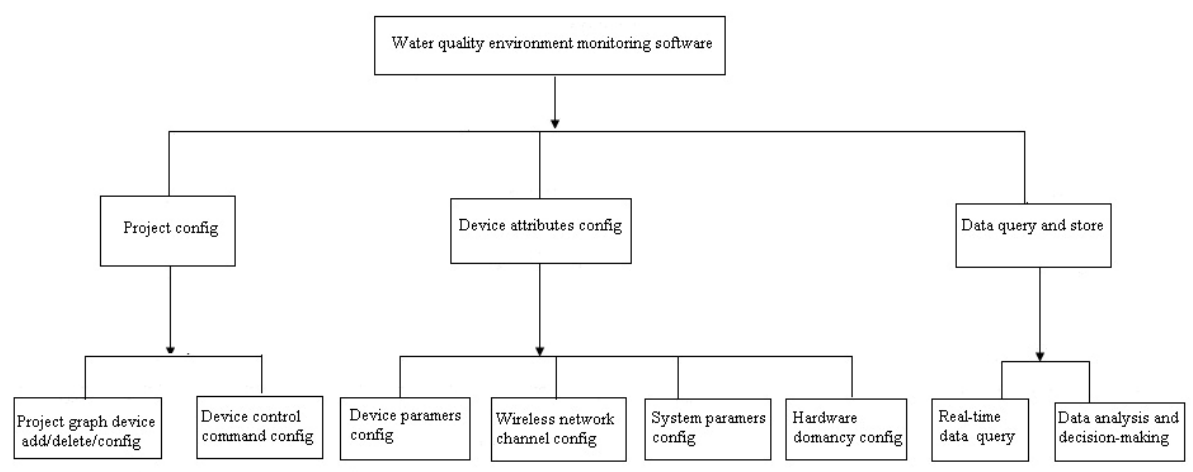

Fig. 4. Software functions structure

Project design module. The functions in this module are mainly related to water quality monitoring on-site equipment graphical deployment and configuration. The user can present the equipment deployment in a project in a graphical method. It is easy for users to the project management, and users can design several projects for different demands. Each project can not only be reused, but also convenient and highly interactive to user operation. Friendly user interfaces provide the ability that the users can fully control the state of engineering equipment. Module functions include: 1) Drag the graphical devices to the project to implement the deployment; 2) Configure the graphical devices and associate with the actual physical devices address; 3)The graphical devices in the project allow users to drag and drop, double-click to configure properties, view real-time water quality data in the relation device.

Current popular model of monitoring software is configuration software. The configuration software which has outstanding specialty such as rich user-defined properties, using flexible, and functions powerful, is a software platform tool for data acquisition and control. Its main contents include good man-machine graphical interface, real-time database, real-time control, communications and networking, open data interface, wide support to I/O devices.

As a water quality monitoring software, the configuration of the used devices would help users design and management of the project, so the module uses configuration model on the graphical devices in the development. The graphical devices configuration in the water quality monitoring software include ponds, access node, routing node, collection node, control valves, switches. Users can design the project by dragging and dropping the graphical device from the graphical devices configuration toolbar. All 
graphical devices in the project can be moved, added relations, and viewed the real-time data by clicking in the main project interface. For the collection node and routing node, because they communicate with the software by protocol, the communication state maybe not stabilization in the project. The corresponding graphical device has two states, one green color expresses the good communication state, and the other red color expresses the bad state. Because the communication state refresh in time, the users can manage the device communication state from the project main interface. Also the control device has two state, green expresses the valve open, red expresses close. The control device can be set auto control to implement the operations depend on the monitoring data of the sensor. Users can re-design new projects, and user-designed project can be saved to reuse that implement different management in different projects for the user.

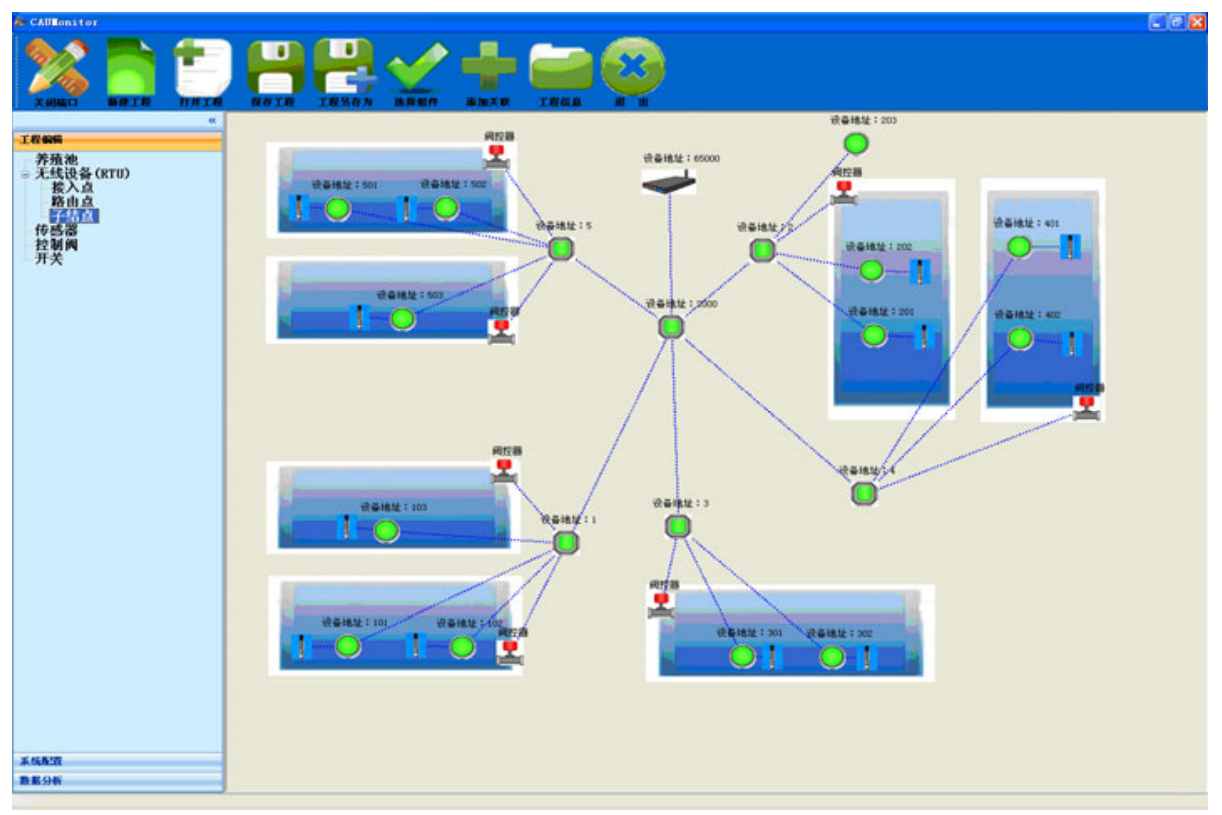

Fig. 5. Project configure interface

Device configure module. The module is mainly related to the physical device information properties, including the address and type of physical device, channel address and type. The functions include: 1) The device attributes configuration; 2) Device-channel attributes configuration; 3) Device parameters configuration, including reading the current configuration and reset the parameters of the device; 4) Device dormant state configuration, including reading the current configuration and reset the device parameters. Device configuration relates to device properties, channel properties, channel parameters, sub-device parameters, system parameters and sleep settings. The device configure main interface shown in Figure 6. 


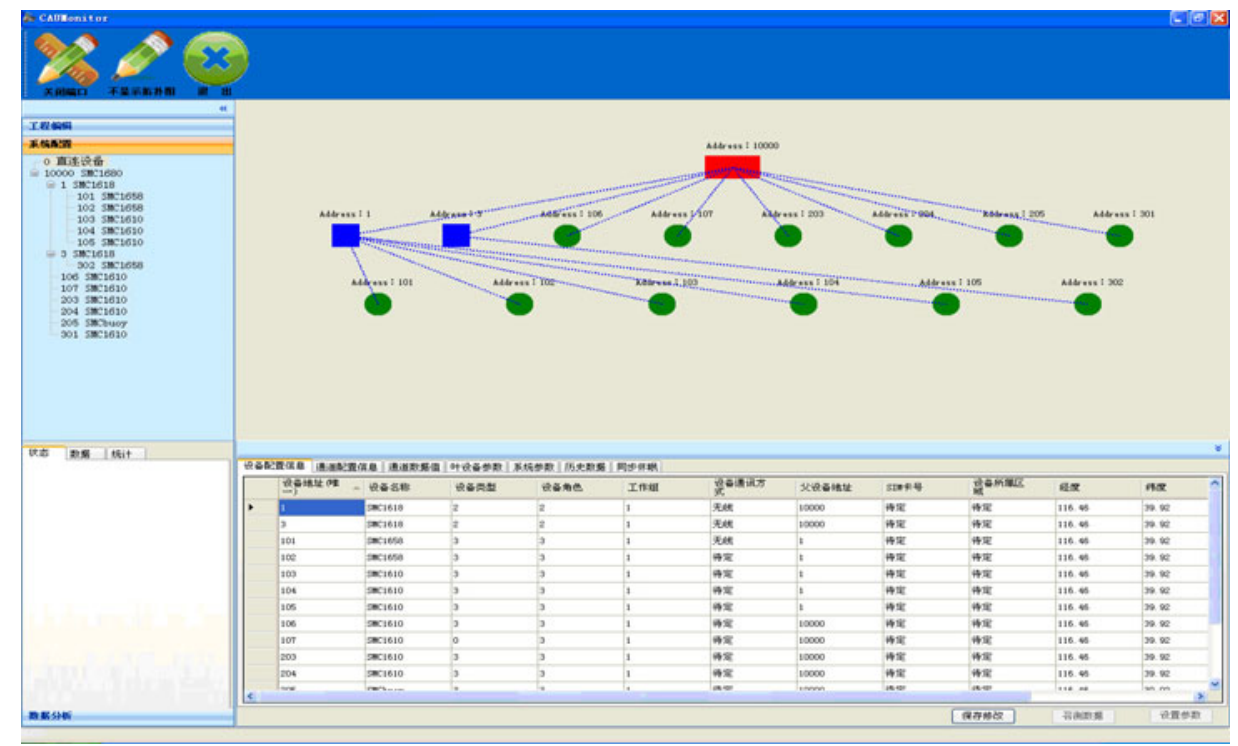

Fig. 6. Device configure interface

Through this module, users can carry out the parameters of the corresponding device configuration management, and water quality monitoring data and device status data will be stored for use. The changes of hardware devices configuration in the wireless sensor network can be completed by the operations that the device properties add, delete, modify in the project. It makes water quality monitoring software to keep devices properties update and have accurate information in time. In the design project module, graphical device associated with the actual hardware device is through adding the device address to the graphical device. In the device configuration module, the device properties and device channel attributes are need to configure fields as shown in Table 1, 2.

Table 1. Device attributes configure data sheet

\begin{tabular}{|c|c|c|c|c|}
\hline $\begin{array}{c}\text { Primary } \\
\text { key }\end{array}$ & Field Name & Field Type & NULL & Description \\
\hline PK & DevAddr & int & $\mathrm{N}$ & Device Address \\
\hline & DevName & varchar & $\mathrm{N}$ & Device Name \\
\hline & DevType & int & $\mathrm{N}$ & Device Type \\
\hline & DevRole & int & $\mathrm{N}$ & Device Role \\
\hline & WorkGroup & int & $\mathrm{N}$ & Work Group \\
\hline
\end{tabular}


Table 1. (continued)

\begin{tabular}{|c|c|c|c|c|}
\hline & $\begin{array}{c}\text { DevComm } \\
\text { Type }\end{array}$ & varchar & $\mathrm{N}$ & $\begin{array}{c}\text { Communication } \\
\text { Type }\end{array}$ \\
\hline & $\begin{array}{c}\text { ParentDev } \\
\text { Addr }\end{array}$ & int & $\mathrm{N}$ & $\begin{array}{c}\text { Parent Device } \\
\text { Address }\end{array}$ \\
\hline & SIM & varchar & $\mathrm{N}$ & SIM Number \\
\hline & Area & varchar & $\mathrm{N}$ & Area \\
\hline & Longitude & int & $\mathrm{N}$ & Longitude \\
\hline & Latitude & int & $\mathrm{N}$ & Latitude \\
\hline & MemoryCell & int & $\mathrm{N}$ & Memory Unit \\
\hline & $\begin{array}{c}\text { InsertTree } \\
\text { View }\end{array}$ & int & $\mathrm{N}$ & Treeview \\
\hline
\end{tabular}

Table 2. Channel attributes configure data sheet

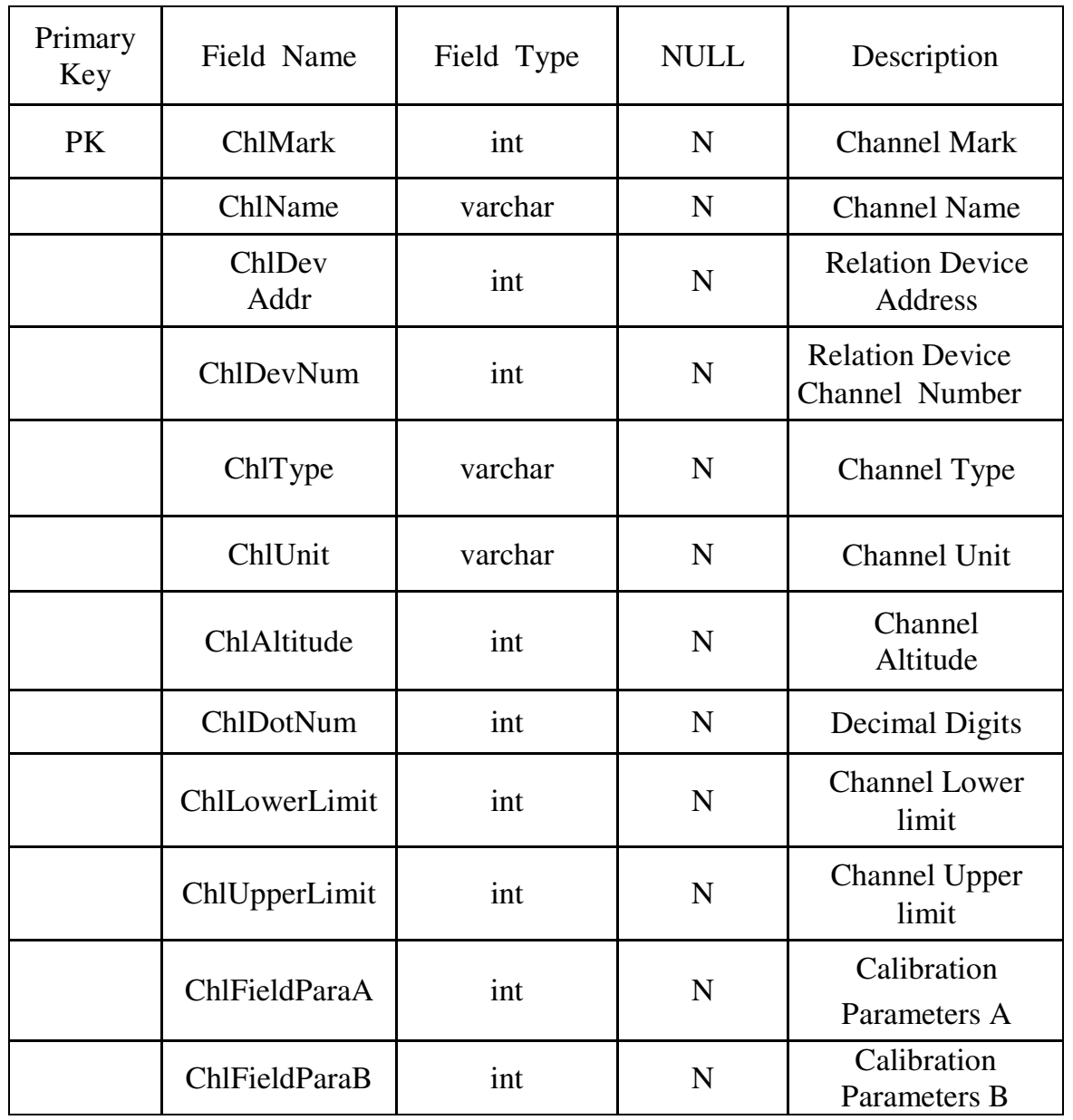


The software can configure the system parameter such as device storage parameters, including the storage of cycles, the begin record time and the end recorded time to adjust the device stored setting. Since sensor devices in the wireless sensor network rely mainly on battery power energy, in order to ensure the device continued work time, device configure module provides a sleep set. Through the sleep setting, users can control the sensor device periodically stop working, and can wake up when needed in a time. Through the setting, it greatly enhances the device working time. In this module, users can also understand the WSN topology of the network, and can get the current device CPU voltage and battery voltage by double-click the graphics device in the topology that facilitates the management of the current device.

Data query/store/analysis module. The module is mainly related to query/store/analysis the monitoring data on the configured device. The functions include: 1) Query and preserve the basic properties of the devices; 2) Query and preserve the basic properties of the device-channel; 3) Query and preserve water quality sensor real-time data; 4)Analyze the graphical device real-time data and make the control command.

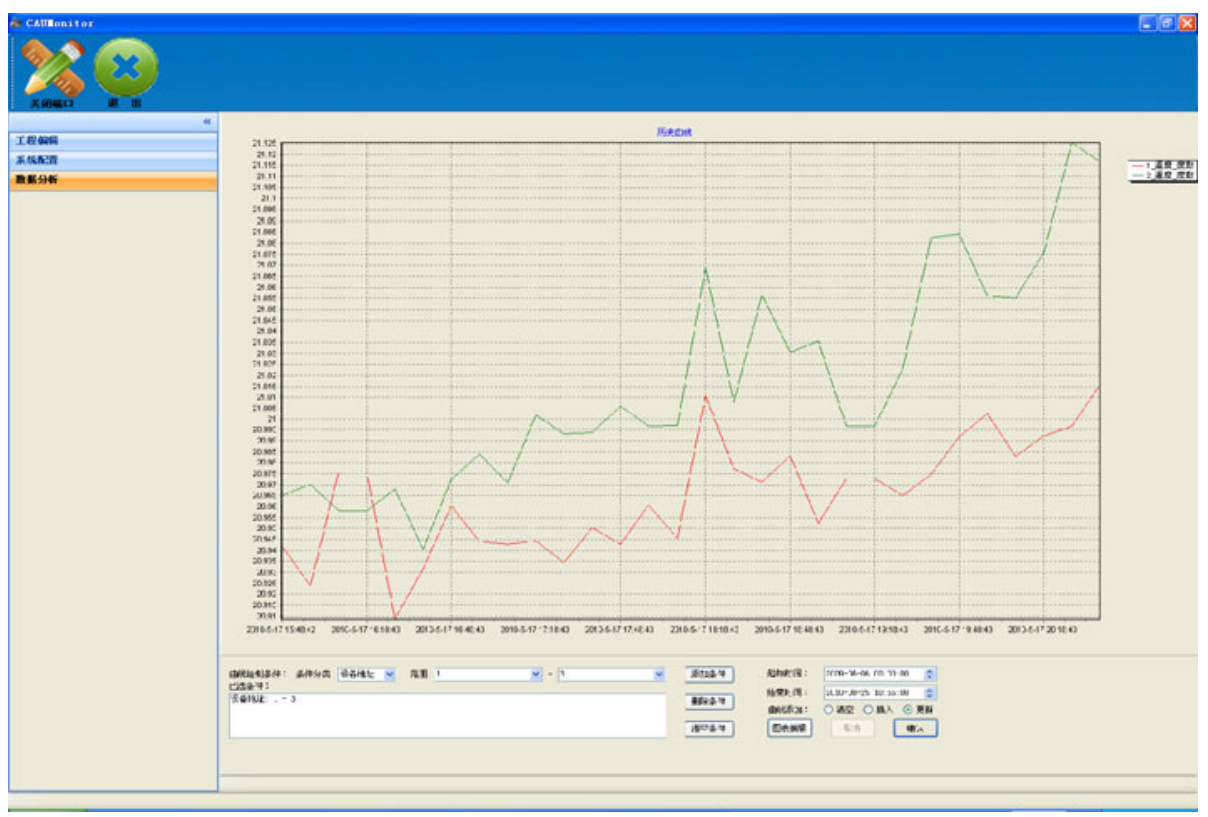

Fig. 7. History data analysis interface

Through the preservation of historical water quality data, the users can analyze the water quality monitoring data, view data trends, and grasp the overall trend of water quality. The data preservation include water quality monitoring data and device status data, and the corresponding data fields shown in Table 3,4. 
Table 3. Water quality data sheet

$\begin{array}{ccccc}\text { Primary } & \text { Field Name } & \text { Field Type } & \text { NULL } & \text { Description } \\ \text { key } & \text { HisData_Index } & \text { int } & \text { N } & \text { Data Index } \\ \text { PK } & \text { RecordTime } & \text { Date } & \text { N } & \text { Record Time } \\ & \text { HisData_ChlSum } & \text { int } & \text { N } & \text { Channel Sum } \\ & \text { HisData_x_x } & \text { int } & \text { N } & \text { Channel Date }\end{array}$

Table 4. Device state data sheet

$\begin{array}{ccccc}\begin{array}{c}\text { Primary } \\ \text { key }\end{array} & \text { Field Name } & \text { Field Type } & \text { NULL } & \text { Description } \\ \text { PK } & \text { HisSts_Index } & \text { int } & \text { N } & \text { Data Index } \\ & \text { RecordTime } & \text { Date } & \text { N } & \text { Record Time } \\ & \text { HisSts_ChlSum } & \text { int } & \text { N } & \text { Channel Sum } \\ & \text { HisSts_x_x } & \text { int } & \text { N } & \text { Channel Date }\end{array}$

Through combined the knowledge in knowledge database with the sensor data in water quality database, the module judged the data whether below the lower limit or higher than the upper limit. If it's true, the graphical device in the project will be twinkled to alarm the user. And the software if judged the device state was auto state, it will auto send commands to the control device. Analysis flow chart is shown in Figure 8.

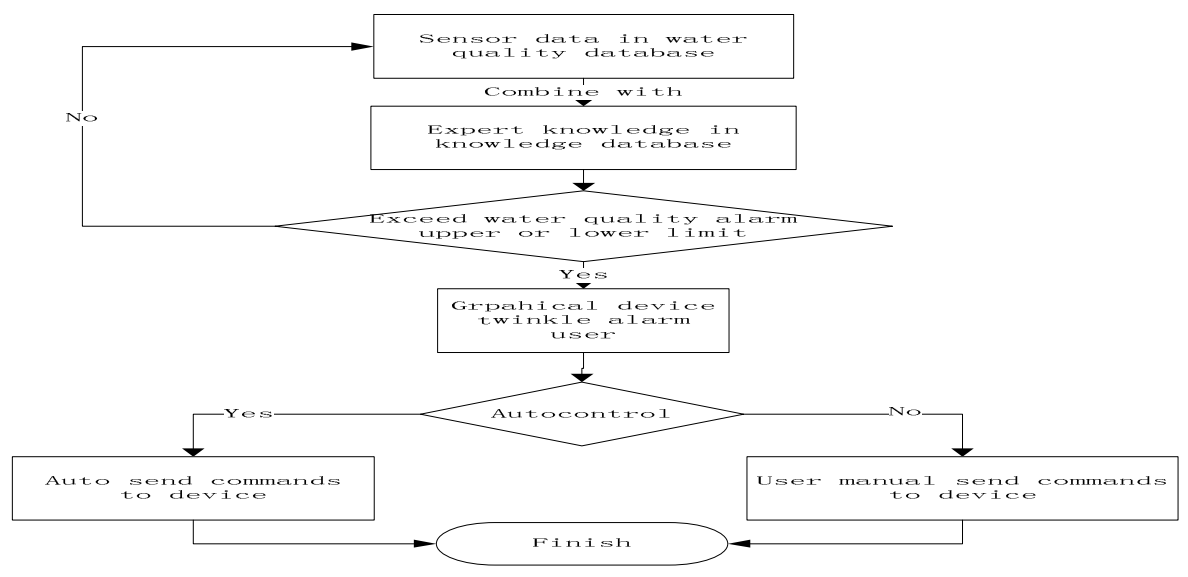

Fig. 8. Analysis flow chart

\section{Application}

The wireless sensor network hardware monitoring equipments deployed in the crab ponds of the local famers in Yixing Jiangsu province. Water quality monitoring software deployed in the control room of PengYao ecological park. The application has received good results in practice. Devices deployment shows as figure 9. 


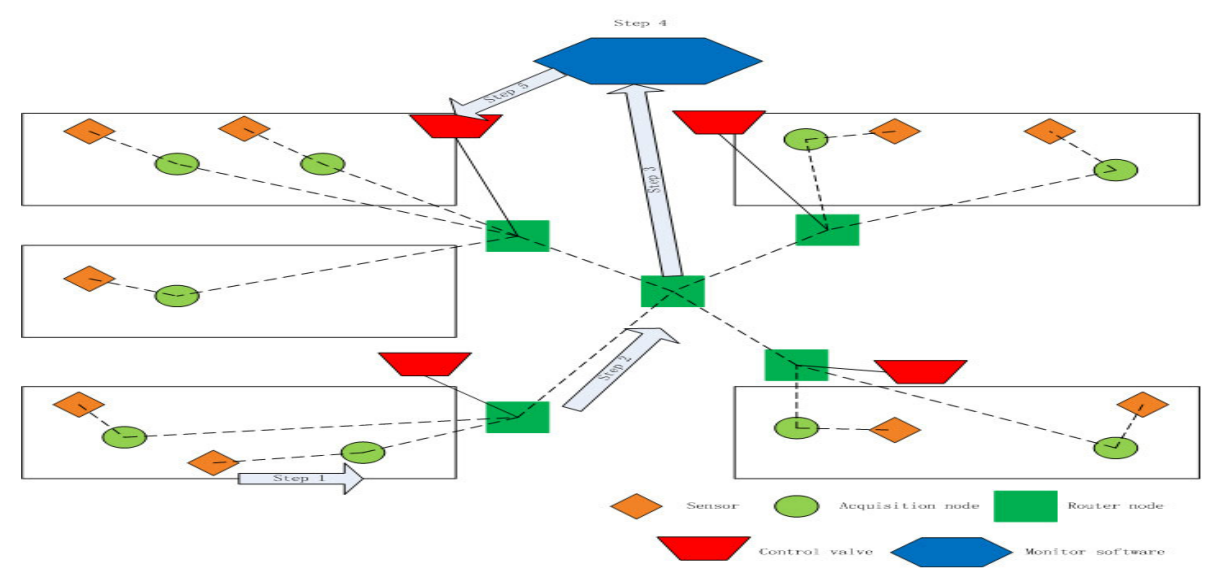

Fig. 9. On-site devices deployment

The steps of monitoring process to the instance of the application are as follows:

(1) Wireless acquisition nodes installed in local farmers' crab ponds, and they are directly connected with the water quality sensors including temperature, water level, PH, DO (dissolved oxygen) sensor to implement the water quality data collection, storage and distribution. Each acquisition node has four sensor interface, can connect up to four sensors to detect five water quality parameters. Acquisition nodes and sensors connected by RS485 communication interface.

(2) Routing nodes installed between the crab ponds and monitoring system center receive data from acquisition node and send to the monitoring system center. Use the routing node can extend the communication distance, and ensure the smooth communication.

(3) The system using ZigBee protocol transmits data to the water quality monitoring system for the related decision-making.

(4) The monitoring system receives the data including water quality data and the node voltage data. Through the water quality data, monitoring system can manage the water quality environment and decision-making. Through the node voltage data, water quality monitoring system can grasp the current energy situation and communication conditions, facilitate equipment maintenance and management.

(5) Send control commands to the control device, and send messages to the user PDA to notify the user, the user can remote control valve by SMS.

\section{Conclusions}

In this paper, a system capable of water quality monitoring based on WSN is presented. The system has made several achievements in: (1) Unlike some simulators, this system is a practical monitoring application where sensor nodes periodically send the information they got to the software for decision making and analysis. (2) Wireless sensor network ensures the real-time and reliability of the monitoring data, and sleeping 
setting make the sensor devices work longer. (3) Configuration software model enhances the reuse and facility of the monitoring project, and provides user-friendly management to the users. (4) The monitoring software can represent the monitoring hardware and data visualization, and analyze the data with expert knowledge to implement the auto control. Furthermore, it can send warning (Via SMS or graphical device twinkle) messages to relevant users when undesirable events are detected.

Future work will deploy more nodes to provide long-term monitoring. Besides, as the water quality parameters correlation between each other, adding data fusion functions and fuzzy control model, which will greatly improve the accuracy of the system monitoring, it will be the next step in the direction of system development.

Acknowledgements. This research was financially supported by the National High Technology Research and Development Program of China (2007AA10Z238), Beijing Natural Science Foundation (4092024), National Major Science and Technology Project of China (2010ZX03006-006) and 948 Project of China Agriculture Ministry (2010-Z13).

\section{References}

[1] Cao, X., Chen, J., Zhang, Y., Sun, Y.: Development of an integrated wireless sensor network micro-environmental monitoring system. ISA Transactions 47(3), 247-255 (2008)

[2] Mainwaring, A., Polastre, J., Szewczyk, R., et al.: Wireless sensor networks for habitat monitoring. In: ACM WSNA 2002, Atlanta, Georgia (2002)

[3] Steere, D.C., Baptista, A., et al.: Research challenges in environmental observation and forecasting systems. In: Proc. 6th ACM/IEEE MobiCOM, pp. 292-299 (2000)

[4] Chen, D., Zheng, Z., Li, J.: Survey on wireless sensor networks. Computer Measurement\& Control (2004)

[5] Ren, F., Huang, H., Lin, C.: Wireless sensor networks. Journal of software, 1282-1291 (2003)

[6] Wang, P.: Introduction and developing trends of online water quality monitoring system in cultivation. Fishery Modernization (2006)

[7] Du, Z., Xiao, D., Zhou, Y., Ou Yang, G.: Design of water quality monitoring wireless sensor network system based on wireless sensor. Computer Engineering and Design 29(17) (2008)

[8] Wang, Z., Hao, X., Wei, D.: Remote water quality mentoring system based on WSN and GPRS. Instrument Technique and Sensor (2010)

[9] Zhao, J., Song, G., Zhou, C., Wei, J.: Research and application of wireless sensor networks in water quality monitor. Communications Technology 41(04) (2008)

[10] Ye, X., Chen, L., Hu, G.: Application of wireless sensor net works in environment monitor. Computer Measurement\&Control, 155-157 (2004)

[11] Song, D., Chen, Q., Xue, Z., Sun, H., Zhang, J.: Study on water quality monitoring system of multi-points online for seawater industrial fish culturing. Marine Fisheries Research 23(4) (2002)

[12] Han, A., He, Y., Li, J., et al.: Design of acoustic signal acquisition system of stored grain pests based on wireless sensor networks. Transactions of the CSAE, 181-187 (2010) 